\title{
Why meta-analyses on the same topic lead to different conclusions?
}

\author{
Francesca Perego - Giovanni Casazza • \\ Gruppo di Autoformazione Metodologica (GrAM)
}

Received: 23 March 2012/Accepted: 5 May 2012/Published online: 25 May 2012

(C) SIMI 2012

The three meta-analyses on aspirin in primary prevention considered in the cutting edge of this issue provide different conclusions about all-cause mortality.

\section{Box 1: summary of meta-analysis main results}

Berger [1] "There was no significant reduction for myocardial infarction, stroke, ischemic stroke, or allcause mortality." (all-cause mortality: RR 0.94, 95 \% CI $0.89-1.00, p=0.07)$.

Raju [2] "Aspirin prevents deaths, myocardial infarction..." (deaths: RR $0.94 ; 95 \%$ CI, $0.88-1.00$ $p=0.05)$.

Seshasai [3] "Modest, but not significant, reductions were observed for total CHD..., total nonvascular mortality..., and all-cause mortality..." (all-cause mortality: OR 0.94; $95 \%$ CI, 0.88-1.00).

Which are the reasons?

There are at least four sources of variability of the results:

1. the studies included,

2. the extraction of patients from primary studies,

On behalf of GrAM.

F. Perego

Dipartimento di Medicina, Medicina Interna III, Università degli

Studi di Milano, Ospedale "L. Sacco", Milan, Italy

G. Casazza $(\bowtie)$

Dipartimento di Scienze Cliniche "L. Sacco",

Università degli Studi di Milano, Via G.B. Grassi 74,

20157 Milan, Italy

e-mail: giovanni.casazza@unimi.it
3. the definition of the outcomes to analyse,

4. the statistical analysis

Regarding the first item, all the three meta-analyses have included the same nine studies.

Focusing on the second item the number of patients extracted from the nine studies varies among meta-analyses: 102,621 (Berger [1] and Seshasai [3]) and 100,076 (Raju [2]). The authors of the latter meta-analysis excluded 2,545 patients because they were treated with warfarin in addition to aspirin or placebo [4]. Anyway, the results of the three meta-analyses do not seem to be affected by the difference of the patients extracted. Indeed, the three relative risk estimates are 0.94 for all the studies.

Considering the third item the authors identified and defined in the same way the outcome (all-cause mortality).

Finally, statistical analyses slightly differ among studies. All the outcomes are analysed with random effect methods. Two meta-analyses report data as risk ratio (RR), while one as odds ratio (OR). The efficacy measure chosen did not affect the results since the estimates of RR and OR are quite similar. Why? Because when RR approaches 1, the values of $R R$ and $O R$ are quite similar. Otherwise, when $\mathrm{RR}$ is higher/lower than 1, OR overestimates/underestimates RR.

Since none of the previous possible sources of variability of results seems to explain the difference of conclusions, why are the conclusions different? What is the mystery?

Scientific studies should be planned to give the answer to a scientific question. In this example, "is aspirin more effective than placebo in the prevention of cardiovascular events or deaths?". This question is translated into a statistical hypothesis (null hypothesis: aspirin is equal to placebo in the prevention of cardiovascular events or 
deaths). To test the null hypothesis we need to select a sample of patients from a defined population (inclusion/ exclusion criteria) and to estimate a measure of association (for e.g. RR) in the selected sample. Considering the observed sample only, could the null hypothesis be falsified or not? With the help of a statistical test, we reject (aspirin is better than placebo) or do not reject (we cannot conclude that aspirin is better than placebo) the null hypothesis on the basis of the estimated RR in the selected sample.

Usually, the results of hypothesis testing are reported in terms of $p$ value. The $p$ value is an estimate of the probability of obtaining the RR calculated in our sample (or a more extreme one), when aspirin is equal to placebo. In the meta-analysis of Berger, the estimate of RR is 0.94 , with a $p$ value of 0.07 . It means that, if aspirin is equal to placebo $(\mathrm{RR}=1)$, the probability of observing a RR of 0.94 (or lower) is 0.07: the null hypothesis cannot be rejected, since the $p$ value is greater than the cut-off value $(0.05)$. For this reason, the authors cannot conclude that aspirin is better than placebo. Therefore, low $p$ values correspond to strong evidence against the null hypothesis (small $p$ value, aspirin better than placebo) while high $p$ values correspond to weak (or absence of) evidence against the null hypothesis. If the $p$ value is lower than (or equal to) a prespecified value (conventionally fixed at 0.05 ) the null hypothesis is rejected and the results are considered statistically significant. Otherwise, if the $p$ value is greater than 0.05 the null is not rejected. According to this well-established rule, if the $p$ value is 0.049 we have to reject the hypothesis, while if $p=0.051$ we have to accept it. In the meta-analysis of Raju et al., since data reported by authors have been rounded to the second decimal place, $p=0.05$ was $p=0.0455$ (according to Raju et al. [2] Fig. 2, $z=2.00$ ). For this reason the authors conclude that aspirin prevents deaths. The $p$ value indicates a statistical difference between treatment effect (statistical significance) but it does not give us any information about the size and the direction of this difference.

Such a simple statistical hypothesis can also be tested by considering the confidence interval (CI). The CI is a set of values that must be considered equally plausible for the population studied, according to the precision of a sample estimate. It consists of a set of values which you can be confident (with a given confidence level, usually $95 \%$ ) including the true value of RR. Roughly speaking, the $95 \% \mathrm{CI}$ of a RR provides a set of values of the true RR that are highly compatible (with a confidence of $95 \%$ ) with the data collected. Raju et al. report the $95 \%$ CI for all-cause mortality (0.88-1.00): it means that, according to the above definition, all the values between 0.88 and 1.00 are all possible true values for the RR. In this clinical setting, the effect of aspirin may equally be protective with an RR of 0.88 (best scenario) or be not different from placebo $(\mathrm{RR}=1$, worst scenario). Anyway, being $95 \%$ sure to be not wrong, we can exclude that aspirin is less effective than placebo. Moreover, the width of a CI is a measure of the precision of the estimated RR: the narrower the $\mathrm{CI}$, the higher the precision of the estimate (wide $\mathrm{CI}=$ low precision; narrow $\mathrm{CI}=$ high precision). There is a close link between CIs and two-sided $p$ values [5]. It is well known that $\mathrm{RR}=1$ indicates that there is not any effect, while $\mathrm{RR}>1$ or $\mathrm{RR}<1$ indicate that the treatment is associated with the outcome (harm or benefit, respectively). If the $95 \%$ CI contains the RR of 1 (absence of effect), the result is not statistically significant (assuming $p<0.05$ as significant). If the $95 \%$ CI does not include the RR of 1 the result is statistically significant $(p<0.05)$, so CIs give us information about both the strength of the relationship and the statistical significance.

In the three meta-analyses considered, the point estimates of the efficacy of aspirin in primary prevention are exactly the same (OR or $\mathrm{RR}=0.94)$. Regarding the CIs, their width is almost the same. On the basis of $p$ values (when reported) that are slightly different, the studies reached different conclusions. For the first study Aspirin prevents deaths..., for the second There was no significant reduction for... all-cause mortality while for the third Modest, but not significant, reductions were observed for... all-cause mortality. Indeed, these are just different interpretations of the results, but not different results: this becomes clear when you look at the CIs.

In conclusion, even though $p$ values and CIs are usually considered complementary, CIs are much more informative than $p$ values alone, since they are inclusive of the information given by $p$ values and in addition provide information on the precision of the results obtained in a clinical study. If you report the CIs, the reader has all the information needed to evaluate the results in terms of statistical significance and in terms of the precision of the outcome estimate. Therefore, for the above-mentioned reasons, CIs should always be reported in the abstract and in the results section of a scientific paper.

\section{Bottom line for clinicians}

- When you read a scientific paper look for the CIs of the measures of effect (RR) and do not care of the $p$ value: you can guess the statistical significance also by a quick look at the CI limits.

- Look at the lower limit of CI of RR: the true value of $\mathrm{RR}$ could be as low as the lower bound of the CI is.

- Look at the upper limit of CI of RR: the true value of $\mathrm{RR}$ could be as high as the upper bound of $\mathrm{CI}$. 


\section{Conflict of interest None.}

\section{References}

1. Berger JS, Lala A, Krantz MJ, Baker GS, Hiatt WR (2011) Aspirin for the prevention of cardiovascular events in patients without clinical cardiovascular disease: a meta-analysis of randomized trials. Am Heart J 162(1):115-124.e2

2. Raju N, Sobieraj-Teague M, Hirsh J, O'Donnell M, Eikelboom J (2011) Effect of aspirin on mortality in the primary prevention of cardiovascular disease. Am J Med 124(7):621-629
3. Seshasai SR, Wijesuriya S, Sivakumaran R et al (2012) Effect of aspirin on vascular and nonvascular outcomes: meta-analysis of randomized controlled trials. Arch Intern Med 172(3):209-216

4. (1998) Thrombosis prevention trial: randomised trial of lowintensity oral anticoagulation with warfarin and low-dose aspirin in the primary prevention of ischaemic heart disease in men at increased risk. The Medical Research Council's General Practice Research Framework. Lancet 351(9098):233-241

5. Gardner MJ, Altman DG (1986) Confidence intervals rather than $P$ values: estimation rather than hypothesis testing. Br Med J 292 (6522):746-750 